C-A/AP/\#420 Jan. 2011

\title{
Compare 100 GeV/n Au Run 2010 with Run 2007
}

S.Y. Zhang

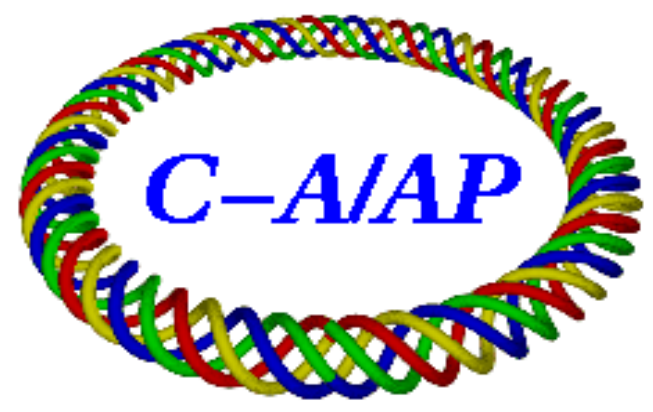

\section{Collider-Accelerator Department}

Brookhaven National Laboratory

Upton, NY 11973

Notice: This document has been authorized by employees of Brookhaven Science Associates, LLC under Contract No. DE-AC02-98CH10886 with the U.S. Department of Energy. The United States Government retains a non-exclusive, paid-up, irrevocable, world-wide license to publish or reproduce the 


\title{
Compare $100 \mathrm{GeV} / \mathrm{n}$ Au Run 2010 with Run 2007
}

\author{
S.Y. Zhang
}

\section{Abstract}

With the very successful commissioning of the vertical stochastic cooling in 100 $\mathrm{GeV} / \mathrm{n}$ Au Run 2010, the IBS (intra-beam scattering) is no longer the dominant factor in terms of the integrated luminosity. A new luminosity model is needed, where the beam intensity lifetime is more important and the burn-off needs to be accounted for. Toward this goal, a brief review of the Run 2010, compared with Run 2007, is presented.

\section{Compare Run 2010 with Run 2007}

Run 2010 and Run 2007 are compared under the following conditions:

1. For Run 2007, using 10 fills with the best integrated luminosity: 8803, 8805, 8808, 8815, 8816, 8825, 8983, 9029, 9030, 9040. All with the longitudinal stochastic cooling in Yellow, but not in Blue.

2. For Run 2010, using 10 fills with the best integrated luminosity: 11821, 11824, $11832,11833,11835,11842,11850,11858,11859,11860$. All with the longitudinal stochastic cooling in Blue, but not in Yellow. Vertical stochastic coolings are applied in both rings, but in a sequential mode, cooling with one then the other [1].

3. Only the beam parameters at 0.5 hour and 3.5 hour after the transition are considered. Note that within this period of time, the integrated luminosity accounts approximately $66 \%$ of the total luminosity, and before 0.5 hour and after 3.5 hour account approximately $17 \%$ each. For complete account of the beam parameters, readers are referred to [2].

4. The decay patterns of the luminosity and the beam intensity are quite different [3]. Moreover, the luminosity decays in 2007 and 2010 are different, and also the intensity decays with and without longitudinal cooling are different. In this note, both the luminosity and beam intensity lifetime is calculated as the linearly averaged one during the 0.5 hour to 3.5 hour after the transition. 
5. The transverse emittance is calculated using the PHENIX ZDC (zero degree calorimeter) and the Blue and Yellow intensity from the DCCT.

Main parameters in Run 2007 and Run 2010 are listed below.

\begin{tabular}{cccc}
\hline \hline Parameter & Unit & Run 2007 & Run 2010 \\
\hline Beta star & $\mathrm{m}$ & 0.8 & 0.7 \\
Bunch number & & 103 & 111 \\
Bunch intensity, @ 0.5 hour & $10^{9}$ ions & 1.07 & 1.12 \\
Transverse emittance, @ 0.5 hour & $\pi \mu m$ & 16.9 & 15.5 \\
Transverse emittance growth rate & $\%$ / hour & 29.8 & 8.2 \\
Blue intensity lifetime & hour & 11.8 & 17.5 \\
Yellow intensity lifetime & hour & 37.7 & 9.8 \\
Luminosity, @ 0.5 hour & $10^{26} \mathrm{~cm}^{-2} \mathrm{~s}^{-1}$ & 23.1 & 34.2 \\
Luminosity, @ 3.5 hour & $10^{26} \mathrm{~cm}^{-2} \mathrm{~s}^{-1}$ & 8.5 & 15.8 \\
Luminosity lifetime & hour & 4.74 & 5.58 \\
\hline \hline
\end{tabular}

Table 1: Main parameters of the $100 \mathrm{GeV} / \mathrm{n}$ Au Run 2007 and Run 2010

Some details are due for explanation.

1. The beta star is from the lattice in Ramp Designer, Au72 for Run 2007 and Au104 for Run 2010 [4], not the measured [2]. All other parameters, below the bunch number in the table, are the averaged one of the best 10 fills in Run 2007 and the best 10 fills in Run 2010.

2. The transverse emittance at the beginning of the collision is similar in Run 2007 and Run 2010, both at approximately $14 \pi \mu m$. At 0.5 hour after the transition, the vertical cooling in Run 2010 has already taking an effect, and hence the emittance is smaller than that in Run 2007, i.e., $15.5 \pi \mu m$ vs. 16.9 $\pi \mu m$. The due improvement of the luminosity is, therefore, from the vertical cooling.

3. There is a fast drop of the luminosity at the early collision, therefore, the luminosity at 0.5 hour is lower than the peak luminosity shown in [2].

Comparison of the Runs 2007 and 2010 is as follows.

1. On the face value, about 35\% luminosity improvement of Run 2010 over Run 2007 is due to the smaller beta star, $14.3 \%$, more bunches, $7.8 \%$, and the higher bunch intensity, $9.6 \%$. 
2. In Run 2007, the transverse emittance growth rate is $29.8 \%$ per hour, indicating that the luminosity lifetime is mostly determined by the IBS caused transverse emittance growth. On the other hand, the emittance growth of 8.2\% per hour in Run 2010 has demonstrated the end of the IBS era in the $100 \mathrm{GeV} / \mathrm{n}$ RHIC Au run with respect to the luminosity lifetime.

3. With the longitudinal cooling, the Blue intensity lifetime in Run 2010 is about a half of the Yellow in Run 2007. Without longitudinal cooling, the Yellow lifetime in Run 2010 is 9.8 hour, therefore, it is the most critical parameter with respect to the integrated luminosity.

\section{Beam intensity lifetime}

In this section, a more detailed look is given for the beam intensity lifetime in Run 2007 and Run 2010. Run 2007 is represented by Fill 8825, which has a best Yellow beam lifetime (with the longitudinal cooling) of 42.5 hours (averaged one is 37.7 hours) and the Blue lifetime of 11.8 hours (averaged one is the same). Run 2010 is represented by Fill 11860, which has a best Blue lifetime (with the longitudinal cooling) of 22.0 hours (averaged one is 17.5 hours) and the Yellow lifetime of 9.9 hours (averaged one is 9.8 hours).

The beam decays are shown in Figure 1, where the burn-off caused decay is also plotted. The burn-off cross section is just set to be 20 times of the ZDC cross section.

1. The beam decay pattern in Run 2007 is rather simple. The Yellow decay is almost all caused by the burn-off, and the Blue decay is mostly due to the longitudinal IBS caused leakage from the RF bucket, which can be well formulated in a tracking [3].

2. The large swing of the Yellow decay in Run 2010 is noticeable: in about half hour of Blue vertical cooling it rises by almost $4 \%$ per hour, then in the next half hour of the Yellow vertical cooling it reduces by the same amount. It is possible that the betatron-synchrotron coupling is playing a role here. The beam-beam parameter is about 0.003 for this fill, not yet an important factor.

3. Perhaps the most important issue in Run 2010 is the large decay during the first 2 hours of the beam collision, for both Blue and Yellow beams. If considering only the Yellow beam decay, one cannot rule out the possibility that this is a longitudinal issue. However, the similarly Blue decay (with the longitudinal cooling) implies that the large decay at the first 2 hours is likely due to the limited momentum aperture. If true, then the relevant issues include the lattice, the working point, and various non-linearity corrections. 
Blue: Blue decay, Red: Yellow decay, Black: Burn-off
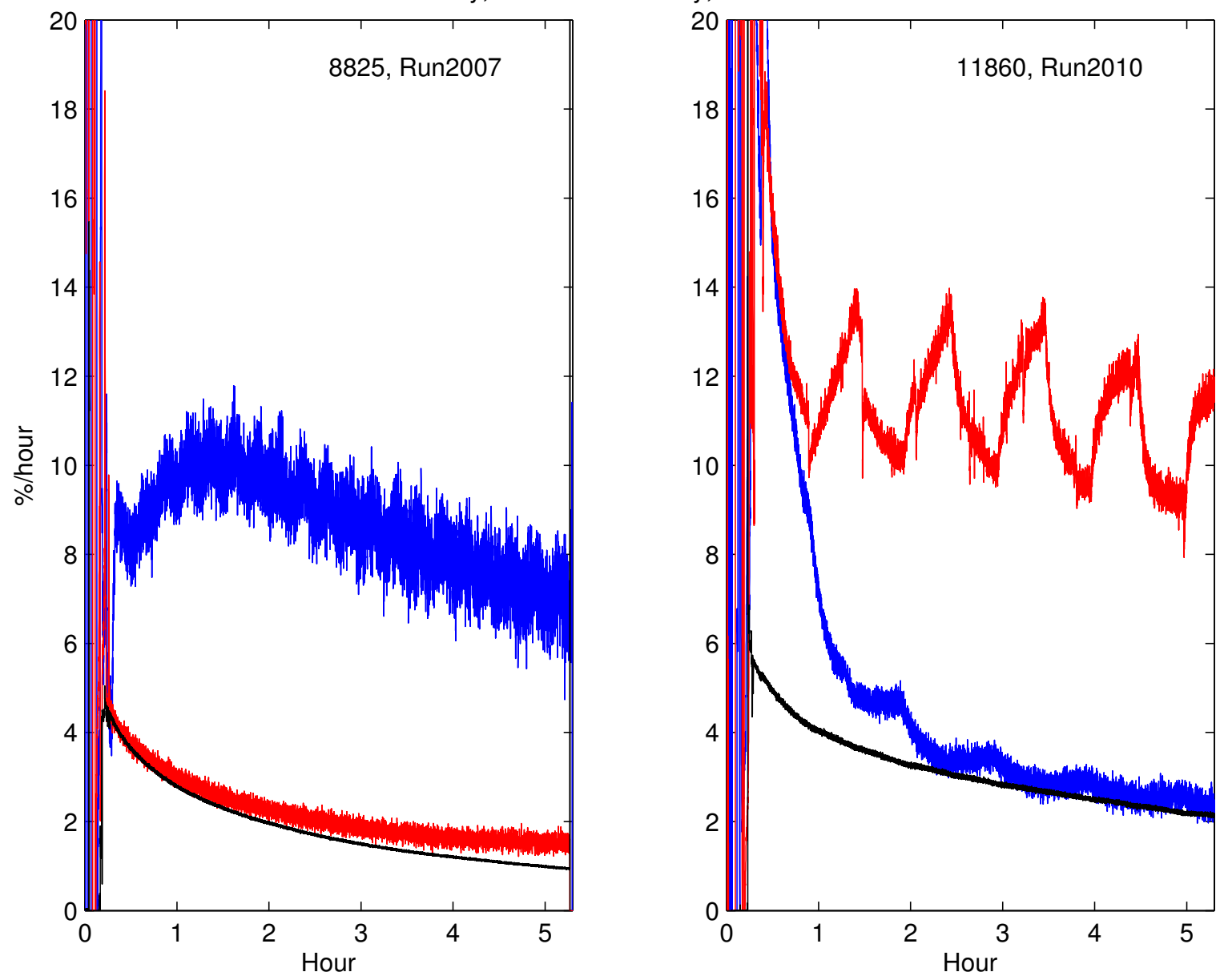

Figure 1: Beam decay of Fill 8825 in Run 2007, and Fill 11860 in Run 2010. The black line shows the decay from the burn-off. 


\section{Luminosity model}

A complete luminosity model for heavy ion runs at both RHIC and LHC is presented in [3]. With tracking and/or solving ODEs (ordinary differential equations), the results agree well with the RHIC Au runs, and the model can be used to predict the luminosity of the LHC lead runs. The model, however, does not include any cooling, either the longitudinal or transverse. As we presented in this note, the vertical cooling applied in RHIC Run 2010 has completely changed the scenario of the integrated luminosity in heavy ion runs. The IBS is no longer the biggest issue. For beam intensity lifetime, Figure 1 shows that the Yellow decay with longitudinal cooling is reduced to almost all from the burn-off in Fill 8825. In [5], it shows that with the vertical cooling, the Yellow emittance is reduced from $20 \pi \mu m$ to $10 \pi \mu m$ in less than 1 hour. The effects of IBS in both longitudinal and transverse dimensions can now be put under control.

In RHIC Au Run 2011, the longitudinal cooling will be applied to both beams, and also the vertical cooling can be applied to both beams simultaneously. The IBS factor will be further reduced from the integrated luminosity, compared with the Run 2010.

A luminosity model with both the longitudinal and transverse stochastic coolings is of interest, which should be benchmarked based on the $100 \mathrm{GeV} / \mathrm{n}$ RHIC Au Run 2011. The main questions addressed by the model might include:

1. The effect of the transverse cooling on the beam intensity and luminosity lifetimes. What is the limit of the transverse cooling?

2. Is there an effect of the beta star on the beam intensity and luminosity lifetimes?

3. The roles of the beam intensity and burn-off on the integrated luminosity.

\section{References}

[1] K.A. Brown et. al., Proceedings of IPAC10, Kyoto, Japan, p.509, 2010

[2] http://www.cadops.bnl.gov/RHIC/Runs/

[3] R. Bruce, J.M. Jowett, M. Blaskiewicz, W. Fischer, Phys. Rev. ST Accel. Beams $13,091001(2010)$

[4] S. Tepikian, private communication.

[5] W. Fischer, Proceedings of IPAC10, Kyoto, Japan, p.1227, 2010 